\title{
Adding flavor on the Higgs branch
}

\author{
Daniel Areán* *1] \\ *Departamento de Física de Partículas, Universidade de Santiago de Compostela \\ and \\ Instituto Galego de Física de Altas Enerxías (IGFAE) \\ E-15782 Santiago de Compostela, Spain
}

\begin{abstract}
We study the holographic dual of the Higgs branch of $\mathcal{N}=4$ SYM in four dimensions coupled to quenched fundamental matter. The fundamentals are added via flavor D7-branes and the Higgs phase is obtained when the color D3-branes and the flavor D7-branes recombine with each other. The holographic dual consists of D7-brane probes in the background generated by the color D3-branes. In this supergravity dual the Higgs phase is realized by switching on a selfdual field strength on the worldvolume of the D7-branes. A complementary microscopic description in terms of dielectric D3-branes is also possible. This microscopic approach permits a better matching with the dual field theory analysis. Finally, the meson mass spectrum is computed by studying the fluctuations of the flavor D7-branes.
\end{abstract}

\footnotetext{
${ }^{1}$ arean@fpaxp1.usc.es
} 


\section{Introduction}

In its original formulation, the AdS/CFT correspondence [1] describes gauge supersymmetric theories with all their fields in the adjoint representation. A natural extension of the duality consists of the inclusion of matter fields in the fundamental representation, which corresponds to the addition of an open string sector to the supergravity side. This approach was initiated in [2, 3, 4] by considering the orthogonal intersection of $N_{f}$ Dq-branes and $N$ Dp-branes with $N_{f} \ll N$. Once the decoupling limit is taken, as $N_{f} \ll N$, the system will consist of $N_{f}$ Dq-brane probes in the background given by the near horizon geometry of $N$ Dp-branes. The open string degrees of freedom living on the Dq-brane probes are identified with the open strings stretching between the Dp- and Dq-branes and thus correspond to fundamental hypermultiplets added to the gauge theory on the worldvolume of the Dp-brane. Moreover, if $q>p$ the decoupling limit forces the $S U\left(N_{f}\right)$ gauge symmetry on the Dq-brane to decouple and this symmetry becomes the global flavor symmetry of the fundamental fields. In this context, the fluctuations of the flavor brane (the Dq-brane) should correspond to mesons in the dual gauge theory. This study was started in [5] for the D3-D7 intersection and it was extended to different probe branes in several backgrounds (for a review see [6, 7]).

The theories dual to the Dp-Dq intersections described above are at the origin of the moduli space. However, one can consider more involved situations such as Higgs phases. Here we will study the SUGRA dual of the Higgs phase for the D3-D7 system. It was proposed in [8, 9] that one can realize a mixed Coulomb-Higgs branch by switching on an instanton configuration of the worldvolume gauge field of the D7-brane. We will give two complementary descriptions of the system: the macroscopic description in terms of the flavor D7-branes and the microscopic one in terms of dielectric D3-branes which become fuzzy along their transverse directions and recombine into the flavor D7-brane. The results presented here are part of a general analysis of the Dp-Dq systems in the Higgs phase published in [10] (see [11] for a review).

\section{D3-D7 intersection}

In this section we will deal with the system given by the intersection of $N$ D3-branes and $N_{f}$ D7-branes, with the D7s being spacetime filling along the D3-branes. When $N_{f} \ll N$ the decoupling limit of the system corresponds to having $N_{f}$ D7-brane probes in the $A d S_{5} \times S^{5}$ geometry generated by D3-branes. It can be seen that the dual gauge theory is $\mathcal{N}=4, d=4$ SYM coupled to $N_{f} \mathcal{N}=2$ fundamental hypermultiplets whose mass is proportional to the separation between the D3- and D7-branes.

The classical SUSY vacua of the dual gauge theory can be obtained by imposing the corresponding $D$ - and $F$-flatness conditions that follow from the lagrangian of the theory. Let us recall that, apart from the $N_{f}$ chiral (antichiral) fundamentals $\mathcal{Q}^{i}\left(\tilde{\mathcal{Q}}_{i}\right)$, the theory has three adjoint hypermultiplets $\Phi_{I}(I=1,2,3)$. We will denote the (scalar) bottom components of the superfields by lowercase letters. The $\phi_{I}$ are the three complex adjoint scalars of $\mathcal{N}=4 \mathrm{SYM}$ and thus they correspond to the transverse scalar fluctuations of the D3-brane. In particular, $\phi_{3}$ represents the fluctuations of the D3 along the plane orthogonal to the flavor D7-brane. Therefore $\Phi_{3}$ is coupled to the fundamentals through a term $\tilde{\mathcal{Q}}_{i}(m+$ 
$\left.\Phi_{3}\right) \mathcal{Q}^{i}$, where $m$ is the mass of the fundamentals, which is proportional to the separation between the D3- and D7-branes.

The $F$-terms corresponding to the fundamental hypermultiplets, vanish by imposing:

$$
\begin{aligned}
& \phi_{3}=\operatorname{diag}\left(\tilde{m}_{1}, \ldots, \tilde{m}_{N-k},-m, \ldots,-m\right), \\
& q^{i}=\left(0 \ldots 0, q_{1}^{i} \ldots, q_{k}^{i}\right)^{T}, \quad \tilde{q}_{i}=\left(0 \ldots 0, \tilde{q}_{i}^{1} \ldots, \tilde{q}_{i}^{k}\right),
\end{aligned}
$$

where the number of $m \mathrm{~s}$ in (1) is $k$ and thus in order to have $\phi_{3}$ in the Lie algebra of $S U(N)$, one must have $\Sigma_{j=1}^{N-k} \tilde{m}_{j}=k m$. As the quark VEV in this solution has some components which are zero and others that are different from zero, this choice of vacuum leads to a mixed Coulomb-Higgs phase. Furthermore, the vanishing of the $F$-terms associated to the adjoint hypermultiplets gives rise to:

$$
\begin{aligned}
& {\left[\phi_{1}, \phi_{3}\right]=\left[\phi_{2}, \phi_{3}\right]=0,} \\
& q^{i} \tilde{q}_{i}+\left[\phi_{1}, \phi_{2}\right]=0,
\end{aligned}
$$

and in view of the vacuum election (2), the matrices $\phi_{1}$ and $\phi_{2}$ in (4) can be considered as $k \times k$ matrices given by the lower $k \times k$ block of the original $N \times N$ matrices. Eq. (4) implies that a non-vanishing VEV of the quark fields $q$ and $\tilde{q}$ induces a non-zero commutator of the adjoint fields $\phi_{1}$ and $\phi_{2}$. These scalars correspond to the four directions transverse to the D3 which lie on the worldvolume of the D7-brane. The dual description in terms of D7-brane probes must therefore involve a non-trivial configuration of the worldvolume gauge field along those directions. Finally, the $D$-flatness condition results in:

$$
\left|q^{i}\right|^{2}-\left|\tilde{q}_{i}\right|^{2}+\left[\phi_{1}, \phi_{1}^{\dagger}\right]+\left[\phi_{2}, \phi_{2}^{\dagger}\right]=0
$$

where again $\phi_{1}$ and $\phi_{2}$ are $k \times k$ matrices. The constraints (3), (4) and (5) define the mixed Coulomb-Higgs phase of the theory.

\subsection{The gravity dual}

There is a one to one correspondence between the Higgs phase of $\mathcal{N}=2$ gauge theories and the moduli space of instantons [12, 13, 14. This follows from the fact that the $F$ and $D$-flatness conditions can be mapped to the ADHM equations (see [15] for a review). Consequently, one can enter the Higgs phase of the gauge theory of the D3-D7 system by adding instantonic DBI flux in the subspace transverse to the D3s (where the gauge theory lives) but contained in the D7-brane, thus naturally realizing the Higgs phase-ADHM equations map.

As we have already said, the gravity dual of the D3-D7 system consists of $N_{f}$ D7-brane probes in the $A d S_{5} \times S^{5}$ geometry sourced by $N$ D3-branes. The metric can be written as:

$$
d s^{2}=\frac{\rho^{2}+\vec{z}^{2}}{R^{2}} d x_{1,3}^{2}+\frac{R^{2}}{\rho^{2}+\vec{z}^{2}}\left(d \vec{y}^{2}+d \vec{z}^{2}\right),
$$

with $R^{4}=4 \pi g_{s} N\left(\alpha^{\prime}\right)^{2}$. The D3-branes lie along the $\mathbb{R}^{1,3}$ given by $d x_{1,3}^{2}$. Notice that the six-dimensional subspace orthogonal to the D3-branes is spanned by the coordinates 
$\vec{y}=\left(y^{1}, \ldots, y^{4}\right)$ and $\vec{z}=\left(z^{1}, z^{2}\right)$, with $\rho^{2}=\vec{y} \cdot \vec{y}$ and $r^{2}=\rho^{2}+\vec{z}^{2}$. Additionally, the SUGRA solution includes a four-form RR potential:

$$
C^{(4)}=\left(\frac{r^{2}}{R^{2}}\right)^{2} d x^{0} \wedge \cdots \wedge d x^{3} .
$$

We shall now embed a stack of $N_{f}$ D7-brane probes in this geometry. Let us take $\xi^{a}=\left(x^{\mu}, y^{i}\right)$ as worldvolume coordinates and consider an embedding in which $|\vec{z}|=L$. $L$ represents the constant transverse separation between the two stacks of D3- and D7-branes and as a consequence, the D3-D7 strings have a mass $L / 2 \pi \alpha^{\prime}$ which corresponds to the quark mass in the field theory dual. In addition, let us assume that the worldvolume field strength $F$ has non-zero entries only along the subspace parametrized by the $y^{i}$ coordinates: $F_{y^{i} y^{j}} \equiv F_{i j}$. It is straightforward to see that for this configurations the DBI and WZ terms of the worldvolume action of the D7-branes take the form:

$$
\begin{aligned}
& S_{D B I}^{D 7}=-T_{7} \int d^{4} x d^{4} y \operatorname{Str} \sqrt{1+\frac{1}{2}\left(\frac{\rho^{2}+L^{2}}{R^{2}}\right)^{2} F^{2}+\frac{1}{16}\left(\frac{\rho^{2}+L^{2}}{R^{2}}\right)^{4}\left({ }^{*} F F\right)^{2},} \\
& S_{W Z}^{D 7}=T_{7} \int d^{4} x d^{4} y \operatorname{Str}\left[\frac{1}{4}\left(\frac{\rho^{2}+L^{2}}{R^{2}}\right)^{2} F F\right],
\end{aligned}
$$

where ${ }^{*} F F={ }^{*} F_{i j} F_{i j}$ and ${ }^{*} F_{i j}=1 / 2 \epsilon_{i j k l} F_{k l}$. Though the entries in (8) and (9) are $S U\left(N_{f}\right)$ matrices, inside the symmetrized trace (Str) they can be considered as commutative numbers.

We shall now consider a configuration in which $F$ is selfdual along the internal $\mathbb{R}^{4}$ spanned by the $y^{i}$ coordinates. In this case one has ${ }^{*} F=F$ and $(8)$ becomes the square root of a perfect square. Consequently, the full action for a self-dual configuration is just:

$$
S^{D 7}(\text { selfdual })=-T_{7} \int d^{4} x d^{4} y \operatorname{Str}[1]=-T_{7} N_{f} \int d^{4} x d^{4} y
$$

Notice that the total action does not depend on $L$. This no-force condition is an explicit manifestation of the SUSY of the system.

The role played by the instantonic worldvolume gauge field can be better understood by recalling that for selfdual configurations the integral of the Pontryagin density $\mathcal{P}(y)$ is quantized for topological reasons:

$$
\int d^{4} y \mathcal{P}(y)=k, \quad \text { with } \quad \mathcal{P}(y) \equiv \frac{1}{16 \pi^{2}} \frac{1}{\left(2 \pi \alpha^{\prime}\right)^{2}} \operatorname{tr}\left[{ }^{*} F F\right],
$$

where $k \in \mathbb{Z}$ is the instanton number. Then, by looking at the WZ term of the action one can check that a worldvolume gauge field satisfying (11) has the effect of inducing $k$ units of D3-brane charge in the D7-brane worldvolume. Finally, it is worth to remark that the existence of these instantonic configurations is possible because we are considering $N_{f}>1$ flavor D7-branes thus having a non-abelian worldvolume gauge theory. 


\subsection{Microscopic description}

In this subsection we will describe the flavor D7-branes with $k$ D3-branes dissolved on them in terms of $k$ dielectric D3-branes [16] expanding to a transverse fuzzy $\mathbb{R}^{4}$ and thus reconstructing the flavor D7-brane. In this microscopic description one has a stack of $k$ D3-branes in the background (6). These D3-branes are extended along the four Minkowski coordinates $x^{\mu}$, whereas the transverse coordinates $\vec{y}$ and $\vec{z}$ must be regarded as the matrix scalar fields $Y^{i}$ and $Z^{j}$, taking values in the adjoint representation of $S U(k)$. Since we are interested in a setup where the D3-branes are localized in the subspace transverse to the would-be D7branes, we must take the scalars $Z^{j}$ to be abelian. The dynamics of the dielectric D3-branes is determined by the Myers dielectric action [16] which is the sum of a DBI and WZ term. For our configuration the DBI term reads:

$$
S_{D B I}^{D 3}=-T_{3} \int d^{4} x \operatorname{Str}\left[\left(\frac{\hat{r}^{2}}{R^{2}}\right)^{2} \sqrt{\operatorname{det}\left(\delta_{i j}-\frac{R^{2}}{\hat{r}^{2}} \theta_{i j}\right)}\right],
$$

where $\hat{r}^{2}$ is the matrix $\hat{r}^{2}=\left(Y^{i}\right)^{2}+Z^{2}$, Str represents the symmetrized trace over the $S U(k)$ indices and we have defined the matrix $\theta_{i j}$ as:

$$
i \theta_{i j} \equiv\left[Y^{i}, Y^{j}\right] /\left(2 \pi \alpha^{\prime}\right) .
$$

Hence $\theta_{i j}$ is antisymmetric in the $i, j$ indices and, as a matrix of $S U(k)$, is hermitian. Reading $C^{(4)}$ from (7), the WZ term of the action can be written as:

$$
S_{W Z}^{D 3}=T_{3} \int d^{4} x \mathbf{S t r}\left[\left(\frac{\hat{r}^{2}}{R^{2}}\right)^{2}\right] .
$$

We shall now assume that $\theta_{i j}$ is selfdual with respect to the $i j$ indices, i.e. $1 / 2 \epsilon_{i j k l} \theta_{k l}=\theta_{i j}$, so that there are only three independent matrices: $\theta_{12}=\theta_{34}, \theta_{13}=\theta_{42}$ and $\theta_{14}=\theta_{23}$. Then, it is not difficult to see that the integrand of 12 becomes the square root of a perfect square, and the action of the system, given by the sum of (12) and (14), reduces to:

$$
S^{D 3}(\text { selfdual })=-\frac{T_{3}}{4} \int d^{4} x \operatorname{Str}\left[\theta^{2}\right]=-\pi^{2} T_{7}\left(2 \pi \alpha^{\prime}\right)^{2} \int d^{4} x \operatorname{Str}\left[\theta^{2}\right],
$$

and we have rewritten the result in terms of the tension of the D7-brane since it will be useful below.

This microscopic description in terms of color D3-branes should match the field theory analysis performed at the beginning of section 2. In particular, we expect that the $F$ - and $D$ flatness conditions of the adjoint scalars are the same as the ones satisfied by the transverse scalars of the dielectric D3-branes. Let us then define the following complex combinations of the $Y^{i}$ matrices:

$$
2 \pi \alpha^{\prime} \phi_{1} \equiv\left(Y^{1}+i Y^{2}\right) / \sqrt{2}, \quad 2 \pi \alpha^{\prime} \phi_{2} \equiv\left(Y^{3}+i Y^{4}\right) / \sqrt{2},
$$

where we have introduced the factor $2 \pi \alpha^{\prime}$ to take into account the standard relation between coordinates and scalar fields in string theory. With these identifications one can easily 
compute the commutators appearing in the equations defining the mixed Coulomb-Higgs phase. Actually, the eqs. (4) and (5) become:

$$
q^{i} \tilde{q}_{i}=\left(\theta_{23}-i \theta_{13}\right) /\left(2 \pi \alpha^{\prime}\right), \quad\left|\tilde{q}_{i}\right|^{2}-\left|q^{i}\right|^{2}=\theta_{12} /\left(\pi \alpha^{\prime}\right),
$$

where we have already taken into account the selfduality of $\theta_{i j}$. Furthermore, the adjoint scalar $\phi_{3}$ is proportional to $Z^{1}+i Z^{2}$ and remember that $Z^{1}$ and $Z^{2}$ are abelian, reflecting the fact that the D3-branes are localized in these directions. Hence one has $\left[\phi_{1}, \phi_{3}\right]=\left[\phi_{2}, \phi_{3}\right]=0$ and the constraint (3) is then fulfilled. Notice that eq. (17) provides an identification between the $\theta$ s and the VEVs of the fundamentals. Remarkably, (17) is telling us that having a nonzero VEV of the fundamentals is only possible if the transverse scalars $Y^{i}$ of the D3-branes have non-zero commutators, and thus the D3-branes get polarized along these directions.

It would now be interesting to relate the present microscopic description in terms of dielectric color D3-branes to the macroscopic description of subsection 2.1 in terms of flavor D7-branes. To this aim, let us compare the WZ terms of the D7- and D3-branes given by (9) and (14). This motivates a map between $k \times k$ matrices in the microscopic description and functions of the $y$ coordinates in the macroscopic approach. Indeed, a matrix $\hat{f}$ is mapped to the function $f(y)$ according to the following rule:

$$
\operatorname{Str}[\hat{f}] \Longrightarrow \int d^{4} y \mathcal{P}(y) f(y),
$$

where the kernel $\mathcal{P}(y)$ on the right-hand side of $(18)$ is the Pontryagin density defined in (11). Actually, the comparison between both WZ actions tells us that the matrix $\hat{r}^{2}$ is mapped to the function $\vec{y}^{2}+\vec{z}^{2}$. Notice also that when $\hat{f}$ is the unit $k \times k$ matrix and $f(y)=1$ both sides of (18) are equal to the instanton number $k$ (see eq. (11)). Furthermore, the comparison of the complete actions of the D7- and D3-branes, given by 10 and (15) respectively, leads to:

$$
\left(2 \pi \alpha^{\prime}\right)^{2} \operatorname{Str}\left[\theta^{2}\right] \Longrightarrow \int d^{4} y \frac{N_{f}}{\pi^{2}}
$$

Thus, according to the general rule (18), the function corresponding to $\theta^{2}$ is given by:

$$
\left(2 \pi \alpha^{\prime}\right)^{2} \theta^{2} \Longrightarrow \frac{N_{f}}{\pi^{2} \mathcal{P}(y)} \text {. }
$$

This relation confirms the realization of the Higgs phase of the dual theory through the addition of instantonic flux of the worldvolume gauge field to the flavor D7-branes. Notice that $\theta^{2}$ is a measure of the non-commutativity of the adjoint scalars and (20) implies that this non-commutativity is related to the (inverse of the) Pontryagin density of the macroscopic D7-branes. In addition, $\theta$ is related to the VEVs of the fundamentals through (17). Therefore, eq. 20 implies a relation between the quark VEV and the instanton density on the flavor D7-branes. Let us make this correspondence more precise by studying the one-instanton configuration of the $N_{f}=2$ gauge theory on the D7-branes. In the so-called singular gauge the $S U(2)$ gauge field is given by:

$$
\frac{A_{i}}{2 \pi \alpha^{\prime}}=2 i \Lambda^{2} \frac{\bar{\sigma}_{i j} y^{j}}{\rho^{2}\left(\rho^{2}+\Lambda^{2}\right)}, \quad \text { with } \quad \bar{\sigma}_{i j}=\frac{1}{4}\left(\bar{\sigma}_{i} \sigma_{j}-\bar{\sigma}_{j} \sigma_{i}\right), \quad \sigma_{i}=\left(i \vec{\tau}, 1_{2 \times 2}\right),
$$


where $\vec{\tau}$ are the Pauli matrices (and $\bar{\sigma}_{i}=\sigma_{i}^{\dagger}$ ), $\rho^{2}=\vec{y} \cdot \vec{y}$ and $\Lambda$ is a constant (the instanton size). Computing $F_{i j}$ and inserting it into the expression for $\mathcal{P}(y)$ given in (11) one arrives at:

$$
\mathcal{P}(y)=\frac{6}{\pi^{2}} \frac{\Lambda^{4}}{\left(\rho^{2}+\Lambda^{2}\right)^{4}} .
$$

It can be easily checked that (22) satisfies (11) with $k=1$. This result can shed light on the relation between the Higgs mechanism of the field theory and the instanton density in the holographic description. For simplicity we will assume that all quark VEVs are proportional to some scale $v: q, \tilde{q} \sim v$. Hence (17) implies $\theta \sim \alpha^{\prime} v^{2}$, and plugging this last result and (22) into 20 one gets the following relation:

$$
v \sim \frac{\rho^{2}+\Lambda^{2}}{\alpha^{\prime} \Lambda}
$$

where $\rho$ should be regarded as the energy scale of the gauge theory. In the far IR $(\rho \approx 0)$ (23) reduces to:

$$
v \sim \Lambda / \alpha^{\prime}
$$

which, up to numerical factors, is precisely the relation between the quark VEV and the instanton size that has been obtained in [9]. As for the full expression (23), notice that for any finite non-zero $\rho$ the quark VEV never vanishes and goes to infinity in both the large and small instanton limits. However, in the far IR the quark VEV goes to zero in the small instanton limit. This region should be clearly singular because a zero quark VEV would correspond to an unhiggsed theory and thus extra light degrees of freedom must appear.

\subsection{Fluctuations}

Finally, we will determine the meson spectrum by studying the fluctuations around the instanton configuration described in subsection 2.1. Actually, we will focus on the fluctuations of the worldvolume gauge field $A=A^{\text {inst }}+a$. Then, the total field strength on the D7-brane worldvolume is $F_{a b}=F_{a b}^{\text {inst }}+f_{a b}$ ( $a, b$ run over the worldvolume), $F_{a b}^{\text {inst }}$ is the selfdual field strength of $A^{\text {inst }}$ and $f_{a b}$ is given by:

$$
f_{a b}=\partial_{a} a_{b}-\partial_{b} a_{a}+\left[A_{a}^{i n s t}, a_{b}\right] /\left(2 \pi \alpha^{\prime}\right)+\left[a_{a}, A_{b}^{i n s t}\right] /\left(2 \pi \alpha^{\prime}\right)+\left[a_{a}, a_{b}\right] /\left(2 \pi \alpha^{\prime}\right) .
$$

We shall consider the DBI and WZ terms of the action for a stack of D7-branes with worldvolume coordinates $\xi^{a}=\left(x^{\mu}, y^{i}\right)$, an embedding $|\vec{z}|=L$ (as in subsection 2.1) and with the worldvolume gauge field strength $F_{a b}$ just described. The mass spectrum will be determined by the equation of motion resulting from the action expanded up to quadratic order in the fluctuations.

Let us concentrate on the $N_{f}=2$ one-instanton configuration described above and restrict ourselves to fluctuations with $a_{i}=0$, i.e. those with non-zero components only along the Minkowski directions. Additionally, we assume that $a_{\mu}^{(l)}=\xi_{\mu}(k) f(\rho) e^{i k_{\mu} x^{\mu}} \tau^{l}$, with $k^{\mu} \xi_{\mu}=0$. This ansatz automatically solves the equation of motion of $a_{i}$. The equation for $a_{\mu}$ reduces to a second order differential equation for $f(\rho)$. This equation depends on $M^{2}=-k^{2}$ and has normalizable solutions for a discrete set of values of $M$ giving rise to a discrete mass spectrum. We compute this spectrum by applying a shooting technique, obtaining the result plotted in fig 1 . 


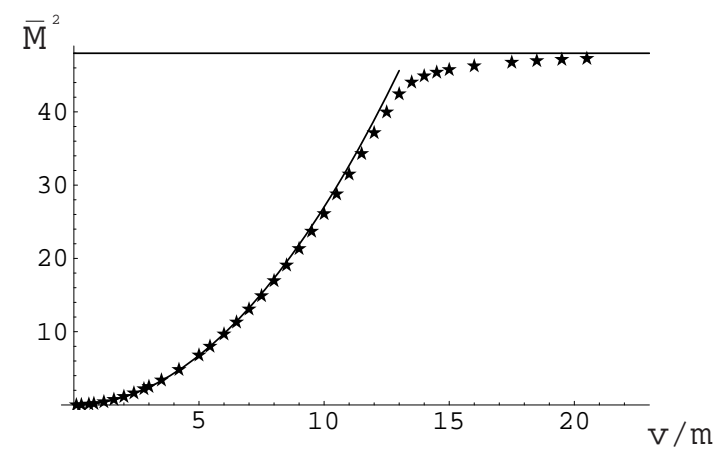

Figure 1: Numerical values of $\bar{M}^{2}=R^{4} L^{-2} M^{2}$ as a function of the ratio of the quark VEV $v=\Lambda /\left(2 \pi \alpha^{\prime}\right)$ and the quark mass $m_{q}=L /\left(2 \pi \alpha^{\prime}\right)$. The solid line represents the prediction obtained by the WKB method. For large $v, \bar{M}$ becomes independent of both $m_{q}$ and $v$ as in [9], while the mass gap vanishes in the small instanton limit $v \rightarrow 0$.

\section{Acknowledgments}

I would like to thank the organizers of the 3rd RTN Workshop (Valencia, October 2007) where this work was presented. The results presented here were obtained in collaboration with Alfonso V. Ramallo and Diego Rodríguez Gómez. I am grateful to Alfonso V. Ramallo and Jonathan P. Shock for useful discussions. This work was supported in part by MEC and FEDER under grant FPA2005-00188, by the Spanish Consolider-Ingenio 2010 Programme CPAN (CSD2007-00042), by Xunta de Galicia (Consellería de Educación and grant PGIDIT06PXIB206185PR) and by the EC Commission under grant MRTN-CT-2004005104.

\section{References}

[1] J. M. Maldacena, Adv. Theor. Math. Phys. 2 (1998) 231, hep-th/9711200.

[2] A. Karch and L. Randall, J. High Energy Phys. 0105 (2001) 008, hep-th/0011156; J. High Energy Phys. 0106 (2001) 063, hep-th/0105132.

[3] A. Karch and E. Katz, J. High Energy Phys. 0206 (2002) 043, hep-th/0205236.

[4] A. Karch, E. Katz and N. Weiner, Phys. Rev. Lett. 90 (2003) 091601, hep-th/0211107.

[5] M. Kruczenski, D. Mateos, R. Myers and D. Winters, J. High Energy Phys. 0307 (2003) 049, hep-th/0304032.

[6] A. V. Ramallo, Mod. Phys. Lett. A21 (2006) 1, hep-th/0605261.

[7] J. Erdmenger, N. Evans, I. Kirsch and E. Threlfall, arXiv:0711.4467 [hep-th].

[8] Z. Guralnik, S. Kovacs and B. Kulik, J. High Energy Phys. 0503, 063 (2005), hepth/0405127. 
[9] J. Erdmenger, J. Grosse, Z. Guralnick, J. High Energy Phys. 0506 (2005) 052, hepth/0502224.

[10] D. Areán, A. V. Ramallo and D. Rodríguez-Gómez, JHEP 0705 (2007) 044, hepth/0703094.

[11] D. Rodríguez-Gómez, Int. J. Mod. Phys. A 22 (2007) 4717, arXiv:0710.4471 [hep-th].

[12] M. R. Douglas, hep-th/9512077.

[13] M. R. Douglas, J. Geom. Phys.28, 255 (1998), hep-th/9604198.

[14] E. Witten, J. Geom. Phys.15, 215 (1995), hep-th/9410052.

[15] D. Tong, hep-th/0509216.

[16] R. C. Myers, J. High Energy Phys. 9912 (1999) 022, hep-th/9910053. 